\title{
Engagement in Practice: Toy Adaptation for Children with Disabilities: En- gaging the Community through Educational Outreach and Toy Donation
}

\author{
Molly Y. Mollica, University of Washington
}

Molly Mollica earned her BS in Biomedical Engineering and her MS in Mechanical Engineering from Ohio State University. She is currently a $\mathrm{PhD}$ student in the Department of Bioengineering at the University of Washington. Her engineering education research focuses are in service learning, increasing diversity in engineering, and adapting toys for children with diverse abilities. Her bioengineering research focuses are in thrombosis mechanics, mechanobiology, and DNA origami nanotechnology.

Alyssa M. Spomer, University of Washington

Brianna M. Goodwin, University of Washington

Dr. Shawn Israel, University of Washington, Departmet of Rehabilitation Medicine

Shawn Israel, PT, DPT is a pediatric physical therapist and instructor at the University of Washington in the Division of Physical Therapy. She has experience working with individuals with a wide variety of neurological diagnoses across their lifespan and feels strongly that everyone should have access to toys, mobility and their environment to enhance their play skills and social interactions.

\section{Dr. Anat Caspi P.E., University of Washington}

Dr. Anat Caspi is director of the Taskar Center for Accessible Technology housed by the Department of Computer Science and Engineering at the University of Washington. Her research interests are in the areas of ubiquitous computing and data science. Caspi is interested in ways by which collaborative commons and cooperation can challenge and transform computing disciplines, and in particular, translation and deployment of technology to benefit individuals with disabilities.

\section{Dr. Heather A Feldner, University of Washington Department of Rehabilitation Medicine}

Heather Feldner received her BS in Human Biology and Master's degree in Physical Therapy from Marquette University. She has been a practicing pediatric physical therapist for 19 years, and began teaching in the University of Illinois at Chicago's DPT program in 2010. She became a board certified pediatric clinical specialist in 2012, completed her Assistive Technology Certificate from UIC in 2015, and earned her PhD in Disability Studies from UIC in 2016. She joined the University of Washington's Department of Mechanical Engineering as a postdoctoral researcher in September of 2016, and recently transitioned to Assistant Professor in the Department of Rehabilitation Medicine at UW. Heather has a special interest in user-centered design and participatory research, and has been a lab member of the GoBabyGo program, which creates custom safety and accessibility modifications to commercially available battery powered toy ride-on cars for children with disabilities, since 2012. Heather's research focuses on investigating the impact of traditional and alternative mobility technologies on the experiences of people with disabilities and their families, and the direct and indirect influences of physical and social environments, technology design, industry, and disability orientation on those experiences.

\section{Dr. Katherine M. Steele, University of Washington}

Dr. Steele is an associate professor in mechanical engineering at the University of Washington. She received her BS in engineering from the Colorado School of Mines and MS and PhD in mechanical engineering from Stanford University. Her research group is dedicated to designing new tools and techniques to improve human ability through engineering. She also leads AccessEngineering, a program to support and encourage individuals with disabilities to pursue careers in engineering. Dr. Steele previously worked in multiple hospitals as an engineer, including The Children's Hospital of Colorado, Lucille Packard Children's Hospital, and the Rehabilitation Institute of Chicago.

\section{Dr. Dianne Grayce Hendricks, University of Washington}


Dr. Dianne Hendricks is a Lecturer in the Department of Human Centered Design \& Engineering and the Director of the Engineering Communication Program at the University of Washington. She designs and teaches courses involving universal design, technical communication, ethics, and diversity, equity and inclusion. She co-founded HuskyADAPT (Accessible Design and Play Technology), where she mentors UW students in design for local needs experts with disabilities. She also leads STEM outreach activities for the UW community and local K-12 students involving toy adaptation for children with disabilities. Dianne holds a PhD in Genetics from Duke University, and BS in Molecular Biology and BA in Psychology from the University of Texas at Austin. 


\title{
Engagement in Practice: Toy Adaptation for Children with Disabilities: Engaging the Community Through Educational Outreach and Toy Donation
}

\begin{abstract}
Play is critical in cognitive, physical, and social development in childhood. However, many offthe-shelf toys are inaccessible to a diverse population of users with disabilities thereby excluding them from realizing these same benefits. Toy adaptation, a process of installing a universal activation port into battery-operated children's toys, addresses a community need for accessible play technology, as it allows for toys to be activated via alternative switches that better meet the needs of the user. Over the last two years, HuskyADAPT (Accessible Design And Play Technology) at the University of Washington has built a sustainable architecture for toy adaptation and distribution to increase community awareness and improve the accessibility of adapted toys in Washington. This paper describes lessons learned and best practices in engaging the community through toy adaptation in two contexts: 1) education outreach and 2) toy donation to families, clinics, and schools.

\section{Introduction}

Toys are essential for development because through play, children get exposure to novel concepts such as cause and effect, develop communication and motor skills, and gain opportunities in independent play and decision making [1-2]. Adapted toys are generally not available in stores, and specialized websites sell adapted toys for 3-5 times the cost of the non-adapted toy. To address this problem, in recent years community and university-driven toy adaptation has become more prevalent across the country to reduce supply-related barriers surrounding accessible play. Organizations such as HuskyADAPT at the University of Washington (UW), the Toy Adaptation Program at the Ohio State University, and the Adaptive Toy Project at the University of North Florida are

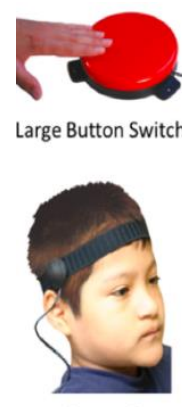

Tilt Switch

Figure 1: Adapted toy and sample activation switches: The dog is an example of an adapted toy with a universal activation port. This adapted toy can be activated by a variety of alternative activation movements including pushing a large button, tilting one's head, pulling a string, biting, or pushing one's jaw downward. This figure is from our previous work [3] and some images are from [4-5].
\end{abstract} working to engage students and community members by teaching toy adaptation adapting and donating accessible toys. Toy adaptation involves modifying electronic toys to make them more accessible to children with diverse abilities. This includes deconstructing a toy and soldering a universal activation port within the toy's circuitry, such that a variety of alternative switches can be used for toy activation (Fig. 1).

Previous studies from other groups have found that toy adaptation is effective in enhancing firstyear engineering students' understanding of the field of engineering, and the connection between engineering and society [6-8]. Additionally, our previous work found that toy adaptation is impactful and engaging to students, community members, and physical therapists [3,9]. Given the greater benefit of toy adaptation in facilitating discussions and training hands on experience in accessible design, we have continued our efforts to engage community members through educational outreach.

Furthermore, over the last two years, HuskyADAPT has built a streamlined process for adapting and donating toys to families, clinics, and schools to serve children with disabilities. The methods 
used contrast those of other organizations [10], given the differences in adapted toy infrastructure between our states. For example, Ohio has forty adapted toy libraries registered with the USA Toy Library Association [11] and Washington has zero (Fig. 2), limiting our ability to distribute toys via existing non-profits and adapted toy libraries as others have described [8]. In response to the dearth of more conventional distribution pipelines, HuskyADAPT has developed alternative solutions to receive and fulfill requests from families, clinics, and schools. Here, we discuss best practices and lessons

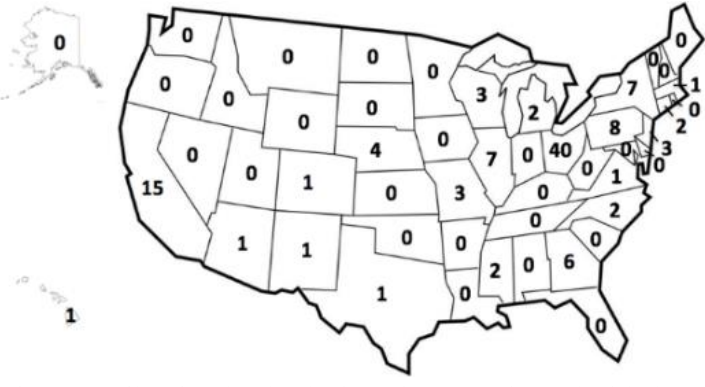

Figure 2: Libraries that lend adapted toys, registered with the USA Toy Library Association [11]. Updated October 2018. learned in establishing both our toy adaptation outreach efforts and our toy donation processes.

\section{Engaging the Community Through Educational Outreach}

\section{Motivation}

We were motivated to include toy adaptation in student based educational outreach efforts based on prior research demonstrating the importance of societal impact [12-13] and the effectiveness of service learning [14-15] to engage underrepresented students in engineering. In our previous work, we surveyed students after they participated in a toy adaptation workshop and found that toy adaptation was well-received, enjoyable, and helped students feel more connected to and see the impact of engineering [9]. For example, when asked what they thought about toy adaptation, one female high school stated, "I think toy adaptation is BRILLIANT and a really beautiful thing. I was AMAZED at how easy it was, and very excited to learn." Because this work is a Community Engagement Division Engagement in Practice paper, it will focus on key logistical issues of methods and lessons learned, but further description and analysis of student responses can be found in our previous work [9].

\section{Methods}

To date, we have engaged over 225 students from 3rd-12th grade in toy adaptation through two methods: 1) on-campus events for students enrolled in summer camps or university programs targeting underrepresented students and 2) classroom events at local schools where toy adaptation has been combined with curriculum on universal design and accessibility.

Partnering with existing university programs to offer on-campus events is a time efficient and affordable way to reach many students, given that there is little or no off-campus travel nor administrative/logistical organization (communication with students, liability waivers, etc.) Examples of previous campus partnerships include the UW Math Academy, the Minority Scholars Engineering Program, Women in Science and Engineering Bridge Program, and Engineering Week. Initial connections with existing on-campus programs occurred through a combination of signup and word of mouth. To manage the multiple requests we received, we found it helpful to launch a Toy Workshop Request Form on our website that collects basic information about event timing, available funding, and number and age of participants. Funding to purchase toys and supplies for these events has occurred through each program's existing budgets, with required supplies averaging $\sim 5 /$ student. HuskyADAPT can reduce costs by maintaining a supply of reusable supplies such as soldering irons and basic hand tools. 
Classroom events at local schools are a valuable means of establishing long-term connections with schools that serve underrepresented students and to deliver more comprehensive curriculum on accessibility and universal design. Connections between HuskyADAPT and interested schools are initially established via the Toy Workshop Request Form, after which we schedule a 2-hour time period for the in-classroom workshop. During events, students first receive a brief lecture introducing toy adaptation, engineering, and universal design, and then work in teams with HuskyADAPT mentors (undergraduate or graduate student volunteers) to modify a toy. We attempt to fulfill as many off campus event requests as possible; however, the popularity of the program has exceeded our current capacity, requiring that we develop creative alternatives. To supplant off campus toy adaptation events, we have found success in inviting teachers to oncampus adaptation events to provide them with the skills and experience necessary to bring the material back into their own classroom. This effort is further supported through our Adapted Toy List [16], an online, open-access repository of photos outlining all toys that we have adapted and specific information regarding their functionality (i.e. lights, sound, motion, bump n' go), their adaptation difficulty rating (easy, medium, or hard) and the specific locations in the toy which were modified. The availability of this document ensures that those with basic familiarity with the toy adaptation process can perform adaptations independently.

Ultimately, we found that toy adaptation is a practical and engaging method for $3^{\text {rd }}-12^{\text {th }}$ grade outreach because it can be accomplished in a short amount of time ( 2 hours) with minimal training, is relatively affordable ( $\$ 5 /$ student), requires only a small collection of portable equipment, and results in the production of a toy immediately ready to be donated.

\section{Lessons learned}

We have found that groups of two students work effectively for toy adaptation. We have observed that larger groups often leave some students unengaged and working individually does not allow students to brainstorm and problem-solve together. We have also found that it is easiest to conduct successful toy adaptation with a trained mentor: ideally, we pair one trained HuskyADAPT mentor with 1-2 groups of inexperienced students. This high ratio allows groups to work at their own pace and engage with the material early in the event by eliminating the need to deliver a long "how-to" introduction. While university students can often conduct toy adaptation in 1.5 hours, 2 or 2.5 hours is a better fit for $3^{\text {rd }}-12^{\text {th }}$ grade students, as it usually takes younger students longer to open their toy, competently use basic hand tools, and learn how to solder. Further, we found that with careful toy selection and the use of wire caps (method to attach two wires without soldering) in place of some soldering, the level of difficulty of toy adaptation can be safely varied to challenge students from $3^{\text {rd }}$ to $12^{\text {th }}$ grade. For example, with $3^{\text {rd }}$ grader groups we often chose to use wire caps, while for high schoolers we will teach them to solder. We can also choose toys with varying circuitry and soldering difficulty, as our Adaptable Toy List provides adequate documentation to aid those familiar with toy adaptation in this selection.

\section{Engaging the Community Through Toy Donation}

\section{Motivation}

In some places, adapted toys are made available through toy lending libraries, which enable community members to freely borrow toys and activation switches. These facilities reduce the financial burden of using accessible toys in care and allow for commitment-free testing to find options that best meet the needs of the child. While these donation channels have proven successful 
for partner organizations, Washington does not currently have any registered toy lending libraries or other formalized means of connecting the community with adapted toys, requiring HuskyADAPT to develop a sustainable distribution pipeline independently.

\section{Methods}

To combat the paucity of adapted toy infrastructure in Washington, HuskyADAPT has developed a publicly available Adapted Toy Request Form [16] to field community donation requests. This form is complemented by the established Adapted Toy List, which allows requesting individuals to preview and select toys that will meet the needs of their children. The request form also facilitates the collection of demographic information, providing HuskyADAPT with a better understanding of the accessibility needs in the area. Requests are filled on a first-come-first serve basis and distributed free of charge to the requesters. Toys that are requested are purchased with a combination of community donations and grants, adapted at biweekly toy adaptation events, and distributed at monthly toy donation drives.

Since going live in July of 2018, HuskyADAPT has received requests from over 50 distinct groups including clinics (15.1\%), schools (46.2\%), families (32.3\%) and other governmental organizations $(6.5 \%)$. On average, 30 toys are requested each month, although this number is steadily climbing as more groups become aware of the program. To date, we have donated over 200 toys to requesters in Western Washington through this program.

The rapid success of the program highlights the obvious demand for accessible toys in Washington; however, as the program is still in its nascency, we have had to make a concerted effort to broadcast the toy donation form to the community. We have solicited requests through three distinct avenues: social media, university and clinical partnerships, and the previously discussed outreach events.

Our website (https://depts.washington.edu/adaptuw) serves as a resource hub and is used to advertise upcoming toy adaptation and distribution events. In addition, we have an active social media presence on Facebook, Twitter, and Instagram which we use to advertise upcoming toy adaptation events, highlight previous events, and connect with a broad demographic including individuals, organizations, and other university programs that work in accessible technology. We also encourage those that receive toys to share their photos on HuskyADAPT platforms and to include links to the Adapted Toy List and Adapted Toy Request Form.

We have also been able to improve awareness for the toy donation program through established connections with university and clinical partners. As HuskyADAPT is a multidisciplinary community of students and faculty across disciplines of engineering, education, and medicine, we have created strong ties throughout the university, establishing effective community partnerships that have facilitated toy donation. Many students that are involved in toy adaptation during their tenure at the university have gone on to be ambassadors for HuskyADAPT in their industries, increasing awareness of the adapted toy program. For example, local clinical partnerships have resulted from students in the Rehabilitation Medicine department sharing their experiences with toy adaptation during clinical rotations. Similarly, partnerships with UW research labs engaging in disability research have provided a direct tie to clinics, families, and organizations in need of switch adapted toys. Additionally, clinicians who have attended adaptation workshops have shared HuskyADAPT's mission and goals with their patients and toys have been provided accordingly.

\section{Lessons Learned}


Implementing our toy donation program has highlighted both difficulties and best practices for establishing and sustaining an accessible toy infrastructure. The primary hurdle for startup was increasing awareness about the program and developing an efficient means of fielding requests. Our multi-modal approach (social media, clinical partnerships, and outreach events) was critical in this process and these efforts remain at the forefront of our attempts to foster a sustainable program. Further, the development of the detailed Adapted Toy List in conjunction with the Adapted Toy Request Form was crucial to empower requesters to make deliberate decisions about their toys while simultaneously allowing us to manage inventory levels based on the types of toys requested.

Early on determining the most appropriate toys to adapt which would be the most engaging and highly requested was another challenge; we relied heavily on expertise from clinical and university partners to select toys that had appropriate functionality based on setting or individual needs. The development of a formalized Adapted Toy Request Form also facilitated data collection on the most requested toys to help guide purchasing and adaptation decisions.

As the program gained more acknowledgement and experienced an influx of requests, inventory management became a primary concern. In order to monitor and fill requests efficiently, an inventory management system was put in place that organized toys by barcode, name, adaptation status (i.e. adapted, unadapted, in repair), and function. The established inventory management system is app-based, allowing for any member from the program to see a real-time snapshot of the available toys and modify entries as toys are donated. This ensures that anyone on the toy donation team can field questions and fill requests efficiently.

An ongoing concern with the toy donation program is establishing best practices for getting toys to requesters. Initially, we allowed individuals to set pickup times; however, this method was not sustainable with scale. We currently host monthly toy donation events which has significantly decreased the demand on the team by concentrating efforts into larger events. Furthermore, this helped improve the efficiency of the donation pipeline, as toys can be requested, purchased, adapted, and donated over the course of a month. This also reduces the demand for a large working inventory, decreasing the space demands of the program. However, as we receive requests from all over Western Washington, the cost of sending the toys is still restrictive. Establishing a financially sustainable means of distributing toys beyond Seattle remains a critical open question for future expansion.

Furthermore, engagement from different stakeholders within our target populations continues to be a concern; we have learned that county-wide efforts and community engagement with stakeholders does not immediately trickle down to individual families. Urban social networks of parents of children with disabilities are often not geographically-based, but rather families often organize in groups related to the disabilities experienced by their children. Better understanding this reality will drive our next phase of community engagement-targeted outreach to specific advocacy organizations and community leaders whom we ask for support.

\section{Conclusions and Future Directions}

Overall, the multifaceted toy adaptation and donation structure that HuskyADAPT has created appropriately meets the unique needs of the region and, while further improvement is necessary to facilitate broader distribution and increased awareness, has dramatically increased the accessibility of adapted toys in Washington. As the toy donation program continues to grow, we are continuing to adapt our structure to ensure that requests are filled expeditiously. We are facilitating more $3^{\text {rd }}$ - 
$12^{\text {th }}$ grade outreach events aimed at teaching students the principles of universal design, accessibility, and the importance of toy adaptation. We are also in the early stages of establishing the first toy lending library in Washington state which will introduce a series of novel logistical challenges ranging from toy maintenance and repair to infection control protocols. Furthermore, we are working to better-integrate the Adapted Toy Request Form and Adapted Toy List [16] into a single web page, allowing individuals to browse photos and videos of toys, see current inventory levels, and submit requests, like many familiar online shopping platforms. We are also continuing to develop improved models for toy donation events to improve the accessibility of adapted toys beyond the Seattle area.

\section{References}

[1] C. Missiuna and N. Pollock, "Play deprivation in children with physical disabilities: The role of the occupational therapist in preventing secondary disability.," American Journal of Occupational Therapy, vol. 45, no. 10, pp. 882-888, 1991.

[2] K. R. Ginsburg, "The importance of play in promoting healthy child development and maintaining strong parent-child bonds," Pediatrics, vol. 119, no. 1, pp. 182-191, 2007.

[3] M. Y. Mollica, H. A. Feldner, A. Caspi, K. M. Steele and D. G. Hendricks, "Toy Adaptation in Undergraduate Education and Outreach: An Initial Examination into Participant Experience and Perceptions," in American Society for Engineering Education, Columbus, Ohio, 2017.

[4] Ablenet, "Switch Accessible Toys," 2017. [Online]. Available: https://www.ablenetinc.com.

[5] EnablingDevices, "Toys For Disabled Children," [Online]. Available: https://enablingdevices.com/catalog/toys_for_disabled_children. [Accessed 2017].

[6] M. Y. Mollica, E. R. Kajfez, M. Riter, M. West and P. Vuyk, "Community Service as a Means of Engineering Inspiration: An Initial Investigation into the Impact of the Toy Adaptation Program," in American Society for Engineering Education, New Orleans, Louisiana, 2016.

[7] R. Kajfez, P. Vuyk, M. Mollica, E. Riter and M. West, "Toy Adaptation Program Workshop: Enriching First-Year Engineers by Teaching the Electronic Toy Adaptation Process," in American Society for Engineering Education, New Orleans, 2016.

[8] M. West, R. L. Kajfez and E. Riter, "One Program's Approach to Creating a Strong Network Paper," in American Society for Engineering Education, Columbus, Ohio, 2017.

[9] M. Y. Mollica, H. A. Feldner, S. Israel, A. Caspi, K. M. Steele and D. G. Hendricks, "Toy Adaptation as Engineering Outreach to Diverse High School Students," in American Society for Engineering Education, Salt Lake City, Utah, 2018.

[10] Toy Adaptation Program, "Ohio State University Toy Adaptation Program (TAP)," [Online]. Available: https://u.osu.edu/osutap/. [Accessed 2019].

[11] USA Toy Library Association, "Toy Library Locations," [Online]. Available: https://www.usatla.org/. [Accessed 2019].

[12] National Academy of Engineers, "Changing the Conversation: Messages for Improving Public Understanding of Engineering," The National Academies Press, Washington, DC, 2008.

[13] Google, "Women Who Choose Computer Science - What Really Matters: The Critical Role of Encouragement and Exposure," 2014.

[14] R. E. Davis, S. Krishnan, T. L. Nilsson and P. F. Rimland, "IDEAS Interdisciplinary Design Engineering and Service International Journal for Service Learning in Engineering, Humanitarian Engineering, and Social Entrepreneurship," pp. 165-179, 2014.

[15] C. Rader, D. Hakkarinen, B. M. Moskai and K. Hellman, "Exploring the appeal of socially relevant computing: are students interested in socially relevant problems?," Proceedings of the 42nd ACM Technical Symposium on Computer Science Education, pp. 423-42.

[16] HuskyADAPT, "HuskyADAPT," [Online]. Available: https://depts.washington.edu/adaptuw. [Accessed 2019]. 